\title{
Nonlinear Optimal Tracking For Missile Gimbaled Seeker Using Finite-Horizon State Dependent Riccati Equation
}

\author{
Ahmed Khamis, D. Subbaram Naidu, and Ahmed M. Kamel
}

\begin{abstract}
The majority of homing guided missiles use gimbaled seekers. The equations describing seeker gimbal system are highly nonlinear. Accurate nonlinear control of the motion of the gimbaled seeker through the attached DC motors is required. In this paper, an online technique for finite-horizon nonlinear tracking problems is presented. The idea of the proposed technique is the change of variables that converts the nonlinear differential Riccati equation to a linear Lyapunov differential equation. The proposed technique is effective for wide range of operating points. Simulation results for a realistic gimbaled system with different engagement scenarios are given to illustrate the effectiveness of the proposed technique.
\end{abstract}

Keywords-Finite-Horizon state dependent Riccati equation, nonlinear tracking, gimbaled missile seeker

\section{INTRODUCTION}

$\mathbf{S}$ TRATEGIC military dependence on missile technology has been growing rapidly since its first use at the beginning of the twentieth century. The critical requirement regarding missile usage is to lead the missile robustly and accurately from its launch point to its designated end point or target. The missile target could be a certain point on its required orbit in space, or a moving hostile object either flying or rolling on terrain. To achieve this requirement, three operations have to be completed and they are described in literature as the Guidance, Navigation and Control (GNC) process [1].

Guidance systems can be categorized into four main categories. These categories are command, beam rider, autonomous, and homing guidance [2]. The majority of homing guided missiles use gimbaled seekers, an example of gimbaled seekers is shown in Fig.1 [2]. The control technique used for the gimbal system on a tactical missile must provide fast and precise tracking of relative error signals created by the missile's signal processing unit. Poor performance during engagement will result in large miss distances which may leads to low probability of mission success. The equations describing the gimbal system under consideration are highly nonlinear. In order to accurately calculate the missile-target LOS angle and its rate, accurate nonlinear control of the motion of the gimbaled seeker through the attached DC motors is required. The linear control techniques become inadequate

Ahmed Khamis and D. Subbaram Naidu are with the Department of Electrical Engineering, Idaho State University, Pocatello, ID, 83209 USA (e-mails: khamahme@isu.edu; naiduds@isu.edu).

Ahmed M. Kamel is with the R\&D department in the Military Technical College (MTC), Cairo, Egypt (e-mail: a_kamel@hotmail.com).

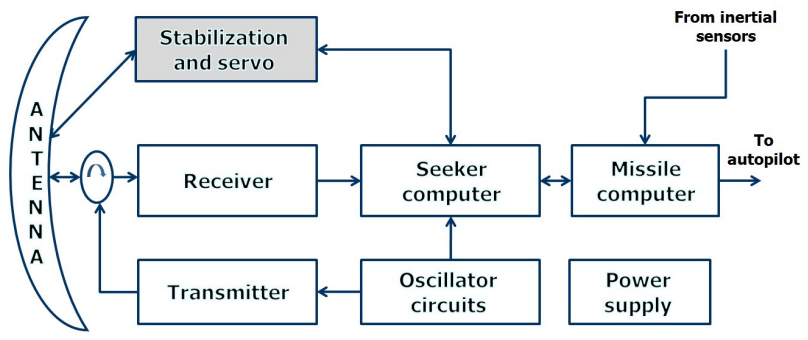

Fig. 1. Active Radar gimbaled Seeker Basic Blocks

and it becomes necessary to use some other nonlinear control techniques. The competitive era of rapid technological change has motivated the rapid development of nonlinear control theory for application to challenging complex dynamical realworld problems [3]. There exist many nonlinear control design techniques, each has benefits and weaknesses. Most of them are limited in their range of applicability, and use of certain nonlinear control technique for a specific system usually demands choosing between different factors, e.g. performance, robustness, optimality, and cost. One of the highly promising and rapidly emerging techniques for nonlinear optimal controllers designing is the State Dependent Riccati Equation (SDRE) technique [4], [5]. Although the SDRE has a great impact in the missile guidance area [6], [7], [8], but none of these works have addressed the problem of finite-horizon optimal control of nonlinear systems.

This paper offers a novel technique for tracking of finitehorizon nonlinear systems. This is accomplished by using the change of variable [9], [10] to convert the nonlinear differential Riccati equation (DRE) to a linear differential Lyapunov equation [11], which can be solved in real time at each time step [12].

The reminder of this paper is organized as follows: The relation between infinite-horizon and finite-horizon SDRE is discussed in Section II. Section III presents the SDRE in finite horizon regulator problem. Section IV presents the nonlinear finite horizon tracking technique via SDRE. Missile system description is presented in Section V. Simulation results are given in Section VI. Finally, conclusions of this paper are given in Section VII.

\section{FINITE-HORIZON SDRE}

Finite-horizon optimal control of nonlinear systems is a challenging problem in the control field due to the complexity 
of time-dependency of the Hamilton- Jacobi-Bellman (HJB) differential equation. In finite-horizon optimal nonlinear control problem, the DRE can not be solved in real time by backward integration from $t_{f}$ to $t_{0}$ because we do not know the value of the states ahead of present time step. To overcome the problem, an approximate analytical approach is used to convert the original nonlinear Ricatti equation to a linear differential Lyapunov equation that can be solved in closed format each time step.

In this section, the relation between the proposed method and the exact optimal solution to the problem will be discussed.

Given the nonlinear system in the form:

$$
\dot{\mathbf{x}}(t)=\mathbf{f}(\mathbf{x})+\mathbf{g}(\mathbf{x}) \mathbf{u}(t) .
$$

The nonlinear system can be expressed in a state-dependent like linear form, as:

$$
\dot{\mathbf{x}}(t)=\mathbf{A}(\mathbf{x}) \mathbf{x}(t)+\mathbf{B}(\mathbf{x}) \mathbf{u}(t),
$$

where $\mathbf{f}(\mathbf{x})=\mathbf{A}(\mathbf{x}) \mathbf{x}(t), \mathbf{B}(\mathbf{x})=\mathbf{g}(\mathbf{x})$.

The exact solution to the optimal control of nonlinear system (2) subject to the cost function

$$
\begin{aligned}
& \mathbf{J}(\mathbf{x}, \mathbf{u})=\frac{1}{2} \mathbf{x}^{\prime}\left(t_{f}\right) \mathbf{F} \mathbf{x}\left(t_{f}\right)+ \\
& \frac{1}{2} \int_{t_{0}}^{t_{f}}\left[\mathbf{x}^{\prime}(t) \mathbf{Q}(\mathbf{x}) \mathbf{x}(t)+\mathbf{u}^{\prime}(\mathbf{x}) \mathbf{R}(\mathbf{x}) \mathbf{u}(\mathbf{x})\right] d t,
\end{aligned}
$$

where $\mathbf{Q}(\mathbf{x})$ and $\mathbf{F}$ is a symmetric positive semi-definite matrix, and $\mathbf{R}(\mathbf{x})$ is a symmetric positive definite matrix, is given by

$$
\mathbf{u}(\mathbf{x}, t)=-\mathbf{R}^{-1}(\mathbf{x}) \mathbf{B}^{\prime}(\mathbf{x})[\mathbf{P}(\mathbf{x}, t) \mathbf{x}(t)+\Pi],
$$

where

$$
\Pi=\frac{1}{2}\left[\begin{array}{lll}
\mathbf{x}^{\prime} \mathbf{P}_{x_{1}} \mathbf{x} & \ldots & \mathbf{x}^{\prime} \mathbf{P}_{x_{n}} \mathbf{x}
\end{array}\right]^{\prime}, \mathbf{P}_{x_{i}}=\frac{\partial \mathbf{P}(\mathbf{x}, t)}{\partial x_{i}} .
$$

$\mathbf{P}(\mathbf{x}, t)$ is a symmetric positive definite solution to the equation

$$
\begin{aligned}
& -\dot{\mathbf{P}}(\mathbf{x}, t)=\mathbf{P}(\mathbf{x}, t) \mathbf{A}(\mathbf{x})+\mathbf{A}^{\prime}(\mathbf{x}) \mathbf{P}(\mathbf{x}, t) \\
& -\mathbf{P}(\mathbf{x}, t) \mathbf{B}(\mathbf{x}) \mathbf{R}^{-1}(\mathbf{x}) \mathbf{B}^{\prime}(\mathbf{x}) \mathbf{P}(\mathbf{x}, t)+\mathbf{Q}(\mathbf{x})+\Omega,
\end{aligned}
$$

with the final condition $\mathbf{P}\left(\mathbf{x}, t_{f}\right)=\mathbf{F}$

where

$$
\Omega=\frac{1}{4} \sum_{i=1}^{n} \sum_{j=1}^{n} \mathbf{P}_{x_{i}} \mathbf{x}\left[\mathbf{B}(\mathbf{x}) \mathbf{R}^{-1}(\mathbf{x}) \mathbf{B}^{\prime}(\mathbf{x})\right]_{i j} \mathbf{x}^{\prime} \mathbf{P}_{x_{j}},
$$

and []$_{i j}$ is the ith element of the $j$ th row of that matrix.

Performing some approximations by neglecting terms $\Omega$ in (6) and $\Pi$ in (4), which leads to the optimal control given by

$$
\mathbf{u}(\mathbf{x}, t)=-\mathbf{R}^{-1}(\mathbf{x}) \mathbf{B}^{\prime}(\mathbf{x}) \mathbf{P}(\mathbf{x}, t) \mathbf{x}(t),
$$

resulted from solving DRE

$$
\begin{aligned}
-\dot{\mathbf{P}}(\mathbf{x})= & \mathbf{P}(\mathbf{x}) \mathbf{A}(\mathbf{x})+\mathbf{A}^{\prime}(\mathbf{x}) \mathbf{P}(\mathbf{x}) \\
& -\mathbf{P}(\mathbf{x}) \mathbf{B}(\mathbf{x}) \mathbf{R}^{-\mathbf{1}}(\mathbf{x}) \mathbf{B}^{\prime}(\mathbf{x}) \mathbf{P}(\mathbf{x})+\mathbf{Q}(\mathbf{x}) .
\end{aligned}
$$

Using these approximations, the control can be approximated to optimal control. The proof is given in [9].

\section{NONLINEAR FINITE-Horizon REgUlator Via SDRE}

Finite-horizon optimal control of nonlinear systems is a challenging problem in the control field due to the complexity of time-dependency of the Hamilton- Jacobi-Bellman (HJB) differential equation. In finite-horizon optimal nonlinear control problem, the DRE can not be solved in real time by backward integration from $t_{f}$ to $t_{0}$ because we do not know the value of the states ahead of present time step. To overcome the problem, an approximate analytical approach is used [9], [10], [11], [12] to convert the original nonlinear Ricatti equation to a linear differential Lyapunov equation that can be solved in closed format each time step.

\section{A. Problem Formulation}

The nonlinear system considered in this paper is assumed to be in the form:

$$
\begin{aligned}
\dot{\mathbf{x}}(t) & =\mathbf{f}(\mathbf{x})+\mathbf{g}(\mathbf{x}) \mathbf{u}(t) \\
\mathbf{y}(t) & =\mathbf{h}(\mathbf{x}) .
\end{aligned}
$$

That nonlinear system can be expressed in a state-dependent like linear form, as:

$$
\begin{aligned}
\dot{\mathbf{x}}(t) & =\mathbf{A}(\mathbf{x}) \mathbf{x}(t)+\mathbf{B}(\mathbf{x}) \mathbf{u}(t), \\
\mathbf{y}(t) & =\mathbf{C}(\mathbf{x}) \mathbf{x}(t),
\end{aligned}
$$

where $\mathbf{f}(\mathbf{x})=\mathbf{A}(\mathbf{x}) \mathbf{x}(t), \mathbf{B}(\mathbf{x})=\mathbf{g}(\mathbf{x}), \mathbf{h}(\mathbf{x})=\mathbf{C}(\mathbf{x}) \mathbf{x}(t)$.

The goal is to find a state feedback control law of the form $\mathbf{u}(\mathbf{x})=-\mathbf{k x}(t)$, that minimizes a cost function given by [13]:

$$
\begin{aligned}
& \mathbf{J}(\mathbf{x}, \mathbf{u})=\frac{1}{2} \mathbf{x}^{\prime}\left(t_{f}\right) \mathbf{F} \mathbf{x}\left(t_{f}\right) \\
& +\frac{1}{2} \int_{t_{0}}^{t_{f}}\left[\mathbf{x}^{\prime}(t) \mathbf{Q}(\mathbf{x}) \mathbf{x}(t)+\mathbf{u}^{\prime}(\mathbf{x}) \mathbf{R}(\mathbf{x}) \mathbf{u}(\mathbf{x})\right] d t,
\end{aligned}
$$

where $\mathbf{Q}(\mathbf{x})$ and $\mathbf{F}$ is a symmetric positive semi-definite matrix, and $\mathbf{R}(\mathbf{x})$ is a symmetric positive definite matrix. Moreover, $\mathbf{x}^{\prime} \mathbf{Q}(\mathbf{x}) \mathbf{x}$ is a measure of control accuracy and $\mathbf{u}^{\prime}(\mathbf{x}) \mathbf{R}(\mathbf{x}) \mathbf{u}(\mathbf{x})$ is a measure of control effort.

\section{B. Solution for Finite-Horizon SDRE Regulator}

To minimize the above cost function (14), a state feedback control law can be given as

$$
\mathbf{u}(\mathbf{x})=-\mathbf{k x}(t)=-\mathbf{R}^{-1}(\mathbf{x}) \mathbf{B}^{\prime}(\mathbf{x}) \mathbf{P}(\mathbf{x}) \mathbf{x}(t),
$$

where $\mathbf{P}(\mathbf{x}, t)$ is a symmetric, positive-definite solution of the State-Dependent Differential Riccati Equation (SDDRE) of the form

$$
\begin{aligned}
-\dot{\mathbf{P}}(\mathbf{x})= & \mathbf{P}(\mathbf{x}) \mathbf{A}(\mathbf{x})+\mathbf{A}^{\prime}(\mathbf{x}) \mathbf{P}(\mathbf{x}) \\
& -\mathbf{P}(\mathbf{x}) \mathbf{B}(\mathbf{x}) \mathbf{R}^{-\mathbf{1}}(\mathbf{x}) \mathbf{B}^{\prime}(\mathbf{x}) \mathbf{P}(\mathbf{x})+\mathbf{Q}(\mathbf{x}),
\end{aligned}
$$

with the final condition

$$
\mathbf{F}=\mathbf{P}\left(\mathbf{x}, t_{f}\right) .
$$

The resulting SDRE-controlled trajectory becomes the solution of the state-dependent closed-loop dynamics

$$
\dot{\mathbf{x}}(t)=\left[\mathbf{A}(\mathbf{x})-\mathbf{B}(\mathbf{x}) \mathbf{R}^{-1}(\mathbf{x}) \mathbf{B}^{\prime}(\mathbf{x}) \mathbf{P}(\mathbf{x})\right] \mathbf{x}(t)
$$


As the SDRE is a function of $(\mathbf{x}, t)$, we do not know the value of the states ahead of present time step. Consequently, the state dependent coefficients cannot be calculated to solve (16) with the final condition (17) by backward integration from $t_{f}$ to $t_{0}$. To overcome the problem, an approximate analytical approach is used [9]. Which converts the original nonlinear Ricatti equation to a differential Lyapunov equation. At each time step, the Lyapunov equation can be solved in closed form. In order to solve the DRE (16), one can follow the following steps at each time step:

1) Solve Algebraic Riccati Equation to calculate the steady state value $\mathbf{P}_{\mathbf{s s}}(\mathbf{x})$

$$
\begin{aligned}
& \mathbf{P}_{\mathbf{s s}}(\mathbf{x}) \mathbf{A}(\mathbf{x})+\mathbf{A}^{\prime}(\mathbf{x}) \mathbf{P}_{\mathbf{s s}}(\mathbf{x}) \\
& -\mathbf{P}_{\mathbf{s s}}(\mathbf{x}) \mathbf{B}(\mathbf{x}) \mathbf{R}^{-1}(\mathbf{x}) \mathbf{B}^{\prime}(\mathbf{x}) \mathbf{P}_{\mathbf{s s}}(\mathbf{x})+\mathbf{Q}(\mathbf{x})=0 .
\end{aligned}
$$

2) Use changing of variables technique and assume that $\mathbf{K}(\mathbf{x}, \mathbf{t})=\left[\mathbf{P}(\mathbf{x}, \mathbf{t})-\mathbf{P}_{\mathbf{s s}}(\mathbf{x})\right]^{-\mathbf{1}}$.

3) Calculate the value of $\mathbf{A}_{\mathbf{c l}}(\mathbf{x})$ $\mathbf{A}_{\mathbf{c l}}(\mathbf{x})=\mathbf{A}(\mathbf{x})-\mathbf{B}(\mathbf{x}) \mathbf{R}^{-1} \mathbf{B}^{\prime}(\mathbf{x}) \mathbf{P}_{\text {ss }}(\mathbf{x})$.

4) Solve the algebraic Lyapunov equation [14]

$$
\mathbf{A}_{\mathbf{c l}} \mathbf{D}+\mathbf{D} \mathbf{A}_{\mathbf{c l}}^{\prime}-\mathbf{B} \mathbf{R}^{-\mathbf{1}} \mathbf{B}^{\prime}=\mathbf{0}
$$

5) Solve the differential Lyapunov equation

$$
\begin{array}{r}
\dot{\mathbf{K}}(\mathbf{x}, \mathbf{t})=\mathbf{K}(\mathbf{x}, \mathbf{t}) \mathbf{A}_{\mathbf{c l}}^{\prime}(\mathbf{x})+\mathbf{A}_{\mathbf{c l}}(\mathbf{x}) \mathbf{K}(\mathbf{x}, \mathbf{t}) \\
-\mathbf{B}(\mathbf{x}) \mathbf{R}^{-\mathbf{1}} \mathbf{B}^{\prime}(\mathbf{x}) .
\end{array}
$$

The solution of (21), as shown by [15], is given by

$$
\mathbf{K}(\mathbf{t})=\mathbf{e}^{\mathbf{A}_{\mathbf{c l}}\left(\mathbf{t}-\mathbf{t}_{\mathbf{f}}\right)}\left(\mathbf{K}\left(\mathbf{x}, \mathbf{t}_{\mathbf{f}}\right)-\mathbf{D}\right) \mathbf{e}^{\mathbf{A}_{\mathbf{c l}}{ }^{\prime}\left(\mathbf{t}-\mathbf{t}_{\mathbf{f}}\right)}+\mathbf{D} .
$$

6) Calculate the value of $\mathbf{P}(\mathbf{x}, \mathbf{t})$ from the equation

$$
\mathbf{P}(\mathbf{x}, \mathbf{t})=\mathbf{K}^{-\mathbf{1}}(\mathbf{x}, \mathbf{t})+\mathbf{P}_{\mathbf{s s}}(t) .
$$

7) Finally, calculating the value of the optimal control $\mathbf{u}(\mathbf{x}, t)$ as

$$
\mathbf{u}(\mathbf{x}, t)=-\mathbf{R}^{-\mathbf{1}} \mathbf{B}^{\prime}(\mathbf{x}) \mathbf{P}(\mathbf{x}, \mathbf{t}) \mathbf{x}
$$

\section{NONLINEAR Finite-Horizon Tracking Using SDRE}

\section{A. Problem Formulation}

Consider nonlinear system given in (10) and (11), which can be redescibed in the form (12) and (13), Let $\mathbf{z}(t)$ be the desired output.

The goal is to find a state feedback control law that minimizes a cost function given by :

$$
\begin{aligned}
& \mathbf{J}(\mathbf{x}, \mathbf{u})=\frac{1}{2} \mathbf{e}^{\prime}\left(t_{f}\right) \mathbf{F e}\left(t_{f}\right) \\
& +\frac{1}{2} \int_{t_{0}}^{t_{f}}\left[\mathbf{e}^{\prime}(t) \mathbf{Q}(\mathbf{x}) \mathbf{e}(t)+\mathbf{u}^{\prime}(\mathbf{x}) \mathbf{R}(\mathbf{x}) \mathbf{u}(\mathbf{x})\right] d t,
\end{aligned}
$$

where $\mathbf{e}(t)=\mathbf{z}(t)-\mathbf{y}(t)$.

\section{B. Solution for Finite-Horizon SDDRE Tracking}

To minimize the above cost function (25), a feedback control law can be given as

$$
\mathbf{u}(\mathbf{x})=-\mathbf{R}^{-1} \mathbf{B}^{\prime}(\mathbf{x})[\mathbf{P}(\mathbf{x}) \mathbf{x}-\mathbf{g}(\mathbf{x})],
$$

where $\mathbf{P}(\mathbf{x})$ is a symmetric, positive-definite solution of the SDDRE of the form

$$
\begin{aligned}
& -\dot{\mathbf{P}}(\mathbf{x})=\mathbf{P}(\mathbf{x}) \mathbf{A}(\mathbf{x})+\mathbf{A}^{\prime}(\mathbf{x}) \mathbf{P}(\mathbf{x}) \\
& -\mathbf{P}(\mathbf{x}) \mathbf{B}(\mathbf{x}) \mathbf{R}^{-\mathbf{1}} \mathbf{B}^{\prime}(\mathbf{x}) \mathbf{P}(\mathbf{x})+\mathbf{C}^{\prime}(\mathbf{x}) \mathbf{Q}(\mathbf{x}) \mathbf{C}(\mathbf{x}),
\end{aligned}
$$

with the final condition

$$
\mathbf{P}\left(\mathbf{x}, t_{f}\right)=\mathbf{C}^{\prime}\left(t_{f}\right) \mathbf{F C}\left(t_{f}\right) .
$$

The resulting SDRE-controlled trajectory becomes the solution of the state-dependent closed-loop dynamics

$$
\begin{array}{r}
\dot{\mathbf{x}}(t)=\left[\mathbf{A}(\mathbf{x})-\mathbf{B}(\mathbf{x}) \mathbf{R}^{-1}(\mathbf{x}) \mathbf{B}^{\prime}(\mathbf{x}) \mathbf{P}(\mathbf{x})\right] \mathbf{x}(t) \\
+\mathbf{B}(\mathbf{x}) \mathbf{R}^{-\mathbf{1}}(\mathbf{x}) \mathbf{B}^{\prime}(\mathbf{x}) \mathbf{g}(\mathbf{x}),
\end{array}
$$

where $\mathbf{g}(\mathbf{x})$ is a solution of the state-dependent nonhomogeneous vector differential equation

$$
\begin{array}{r}
\dot{\mathbf{g}}(\mathbf{x})=-\left[\mathbf{A}(\mathbf{x})-\mathbf{B}(\mathbf{x}) \mathbf{R}^{-\mathbf{1}}(\mathbf{x}) \mathbf{B}^{\prime}(\mathbf{x}) \mathbf{P}(\mathbf{x})\right]^{\prime} \mathbf{g}(\mathbf{x}) \\
-\mathbf{C}^{\prime}(\mathbf{x}) \mathbf{Q}(\mathbf{x}) \mathbf{z}(\mathbf{x}),
\end{array}
$$

with the final condition

$$
\mathbf{g}\left(\mathbf{x}, t_{f}\right)=\mathbf{C}^{\prime}\left(t_{f}\right) \mathbf{F z}\left(t_{f}\right) .
$$

Similar to Section III, an approximate analytical approach is used and the DRE can be solved in the following steps at each time step:

1) Solve for $\mathbf{P}(\mathbf{x}, \mathbf{t})$ similar to the SDDRE regulator problem in Section III

2) Calculate the steady state value $\mathbf{g}_{s s}(\mathbf{x})$ from the equation

$$
\begin{array}{r}
\mathbf{g}_{\mathbf{s s}}(\mathbf{x})=\left[\mathbf{A}(\mathbf{x})-\mathbf{B}(\mathbf{x}) \mathbf{R}^{-1}(\mathbf{x}) \mathbf{B}^{\prime}(\mathbf{x}) \mathbf{P}_{\mathbf{s s}}(\mathbf{x})\right]^{\prime-1} \\
\mathbf{C}^{\prime}(\mathbf{x}) \mathbf{Q}(\mathbf{x}) \mathbf{z}(\mathbf{x}) .
\end{array}
$$

3) Use changing of variables technique and assume that $\mathbf{K}_{\mathbf{g}}(\mathbf{x}, \mathbf{t})=\left[\mathbf{g}(\mathbf{x}, \mathbf{t})-\mathbf{g}_{\mathbf{s s}}(\mathbf{x})\right]$.

4) Solve the differential equation

$$
\mathbf{K}_{\mathbf{g}}(\mathbf{x}, t)=\mathbf{e}^{-\left(\mathbf{A}-\mathbf{B R}^{-1} \mathbf{B}^{\prime} \mathbf{P}\right)^{\prime}\left(\mathbf{t}-\mathbf{t}_{\mathbf{f}}\right)}\left[\mathbf{g}\left(\mathbf{x}, t_{f}\right)-\mathbf{g}_{\mathbf{s s}}(\mathbf{x})\right] .
$$

5) Calculate the value of $\mathbf{g}(\mathbf{x}, t)$ from the equation

$$
\mathbf{g}(\mathbf{x}, \mathbf{t})=\mathbf{K}_{\mathbf{g}}(\mathbf{x}, \mathbf{t})+\mathbf{g}_{\mathbf{s s}}(\mathbf{x}) .
$$

6) Calculate the value of the optimal control $\mathbf{u}(\mathbf{x}, t)$ as

$$
\mathbf{u}(\mathbf{x}, t)=-\mathbf{R}^{-\mathbf{1}}(\mathbf{x}) \mathbf{B}^{\prime}(\mathbf{x})[\mathbf{P}(\mathbf{x}, t) \mathbf{x}-\mathbf{g}(\mathbf{x}, t)] .
$$

Fig.2 summarized the overview of the process of finitehorizon SDDRE tracking technique 


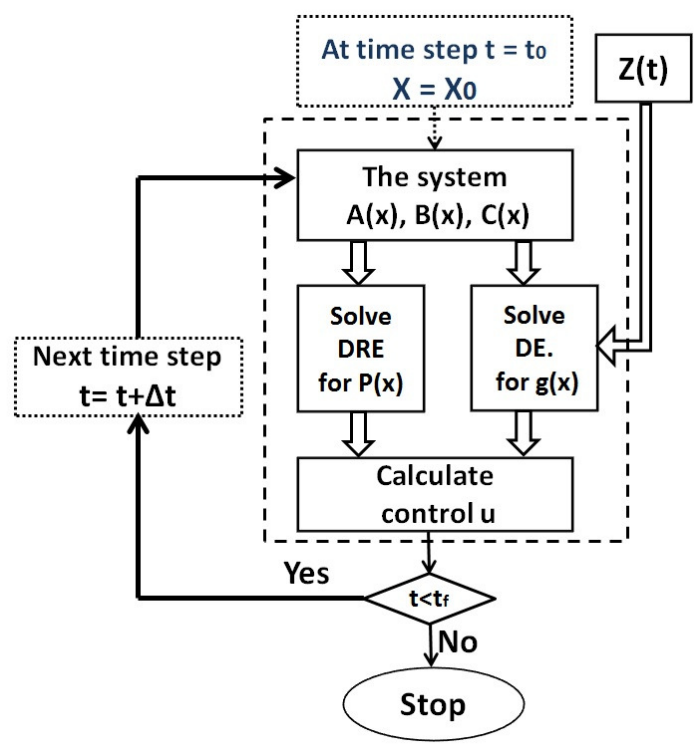

Fig. 2. Overview of The Process of Finite-Horizon SDDER Tracking

\section{Missile System Description}

The missile examined in this paper is a semi active homing guided missile shown in Fig. 3 [16]. The semi-active homing guidance system is based on the principle of utilizing the electromagnetic wave reflection. A ground radar illuminates the target, the missile seeker is designed to home on the reflected energy from the target during its flight. Using this reflected system energy from the target the missile formulates its own correction signals. The missile is steered in space following the proportional navigation guidance method. An on-board guidance kit is utilized to generate the guidance commands. The missile is aerodynamically controlled with an acceleration control autopilot to steer the missile while skid-to-turn (STT) control policy is utilized. The STT steering policy requires two identical lateral (pitch and yaw) autopilots to control the missile attitude while a roll autopilot performs attitude stabilization in the maneuver plane. A roll position controller is utilized to keep an adequate roll damping [17].

The missile flies in air under the effect of thrust, weight, and aerodynamic forces. The action of these forces has a certain effect on the shape of the missile trajectory. The Aerodynamic force is usually distributed to axes of the velocity coordinate system, which are related to the direction of the missile motion. The components of this force are resolved along the missile body axes as $F_{x a}, F_{y a}, a n d F_{z a}$. These forces create aerodynamic moments owing to the fact that they do not pass through the missile center of gravity. The aerodynamic moment components around the missile body axes are $M_{x a}, M_{y a}$, and $M_{z a}$. The missile seeker is a narrow band, continuous wave $(\mathrm{CW})$ receiver that operates as a lock onbefore-launch, semi-active homing system. The system has limited recede capability and cannot lock on targets incoming at less than certain threshold (minimum incoming speed). The missile flight and homing head angles are shown in Fig. 4.

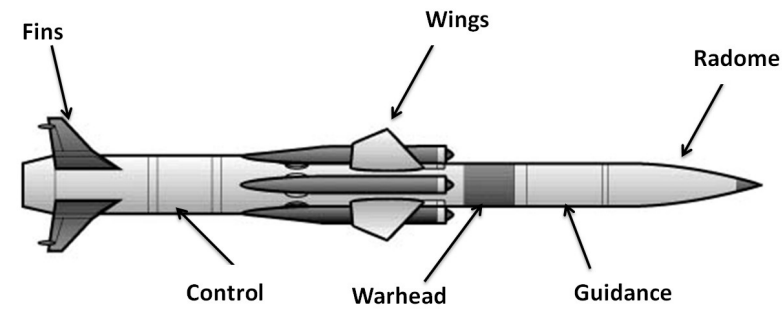

Fig. 3. A Semi Active Homing Missile [16]

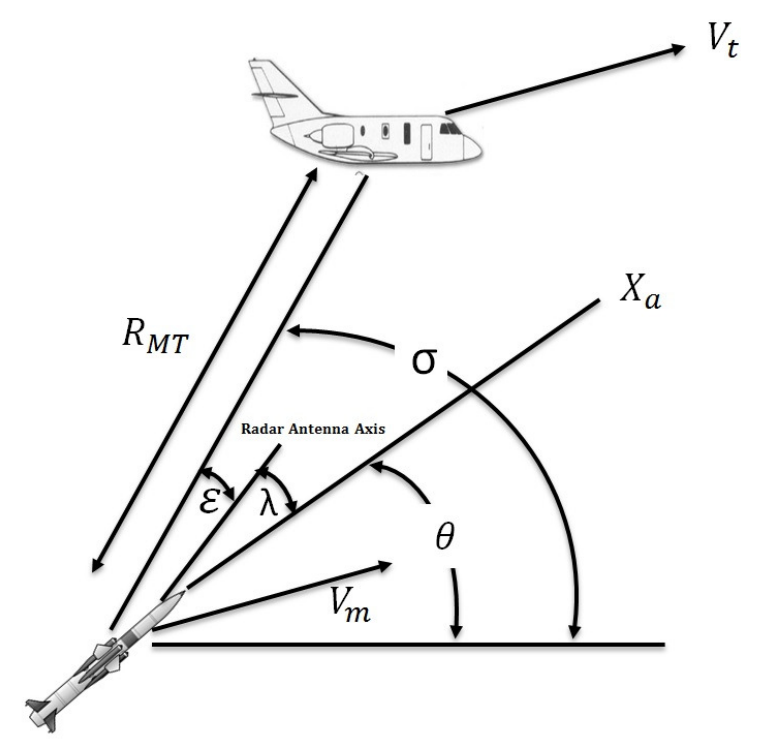

Fig. 4. Missile flight and homing head angles

\section{Simulation Results}

For numerical simulation and analysis, the developed optimal tracking technique in this paper is implemented for a DC motor attached to a realistic gimbaled seeker system. A computer code written under MATLAB environment is employed to solve a missile simulation model [16]. The code is devoted to evaluate the structure of the 6-Degree of Freedom (6 DOF) model in conjunction with the calculation of the desired seeker angles via numerical implementation [18]. Proportional Navigation is the guidance method used in these simulations. In this guidance method, the guidance commands are generated in proportion to the LOS angular rate [19].

Extensive simulation has been carried out. Three engagement scenarios, in pitch plane only for better illustrations, including fixed target, non-maneuvering target, and maneuvering target are considered in the form of case studies.

The dynamic equation for the gimbaled seeker DC motor are:

$$
\begin{aligned}
V(t) & =L \frac{d i(t)}{d t}+R i(t)+k_{b} \frac{d \theta(t)}{d t} \\
m l^{2} \frac{d^{2} \theta(t)}{d t^{2}} & =-m g l \sin (\theta(t))-k_{m} i(t)
\end{aligned}
$$




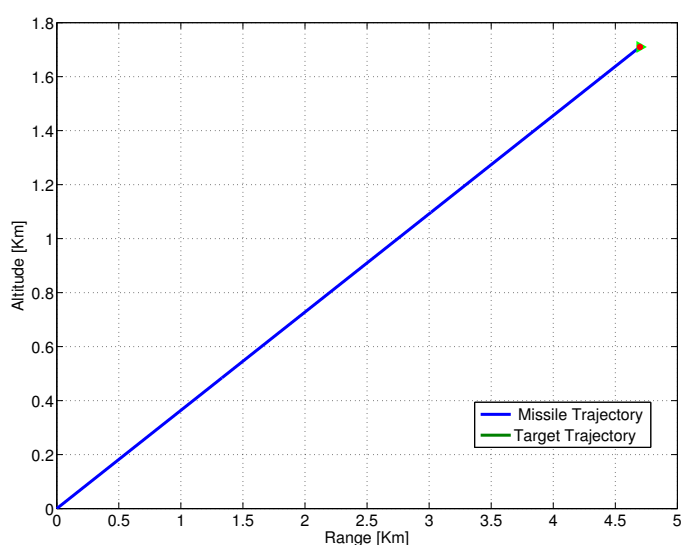

Fig. 5. Missile-Target Engagement Scenario (Case 1)

The system nonlinear state equations can be written in the form:

$$
\begin{aligned}
\dot{x_{1}} & =x_{2}, \\
\dot{x_{2}} & =\frac{g}{l} \sin \left(x_{1}\right)+\frac{k_{m}}{m l^{2}} x_{3}, \\
\dot{x_{3}} & =-\frac{k_{b}}{L} x_{2}+\frac{R}{L} x_{3}+\frac{u}{L}, \\
y & =x_{1},
\end{aligned}
$$

where: $\theta=x_{1}, \dot{\theta}=x_{2}, i=x_{3}, V=u$.

Or alternatively in state dependent form:

$$
\left[\begin{array}{c}
\dot{x_{1}} \\
\dot{x_{2}} \\
\dot{x_{3}}
\end{array}\right]=\left[\begin{array}{ccc}
0 & 1 & 0 \\
\frac{(g / l) \sin \left(x_{1}\right)}{x_{1}} & 0 & \frac{k_{m}}{m l^{2}} \\
0 & -\frac{k_{b}}{L} & \frac{R}{L}
\end{array}\right]\left[\begin{array}{l}
x_{1} \\
x_{2} \\
x_{3}
\end{array}\right]+\left[\begin{array}{l}
0 \\
0 \\
\frac{1}{L}
\end{array}\right] u
$$

The weight matrices are chosen to be

$$
Q=\operatorname{diag}(3000,0,0), R=30, F=\operatorname{diag}(1,1,1) .
$$

\section{A. Case 1: Fixed Target}

Consider a fixed target, in this case the desired seeker angle will be $z(t)=0^{\circ}$, i.e the problem is now a regulator problem.

The simulations were performed for final time of 8 seconds, and the engagement scenario is shown in Fig. 5. The resulting trajectories for the demanded and achieved seeker angles are presented in Fig. 6, the optimal control is shown in Fig. 7, and the optimal error is shown in Fig. 8.

In Fig. 6, the solid line denotes the actual (achieved) angle trajectory of the finite-horizon tracking controller, the dashed line denotes the desired seeker angle.

As shown in Fig. 5, a successful hit is observed. Fig. 6 shows that the finite-horizon SDRE nonlinear regulating algorithm gives excellent results and the developed algorithm is able to solve the SDRE finite-horizon nonlinear regulator problem with a zero average optimal error and $0.003^{\circ}$ standard deviation.

\section{B. Case 2: Non-Maneuvering Target}

Consider a non-maneuvering target (with constant velocity). The simulations were performed for final time of 8 seconds,

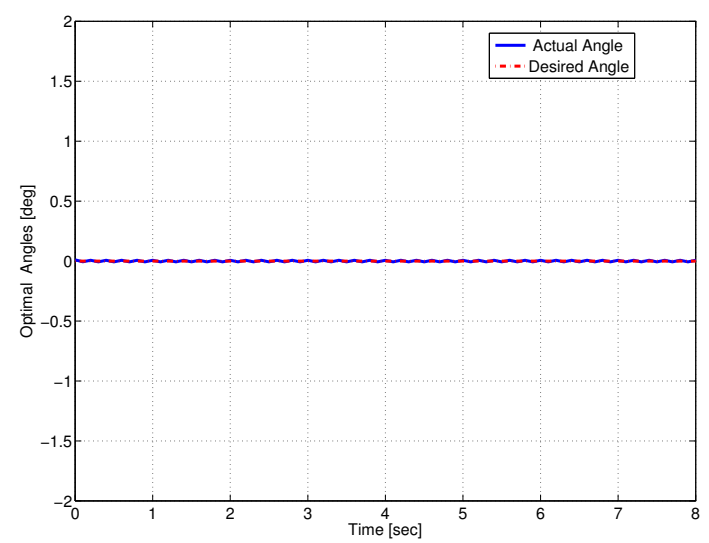

Fig. 6. Angle Trajectories for Gimbaled System (Case 1)

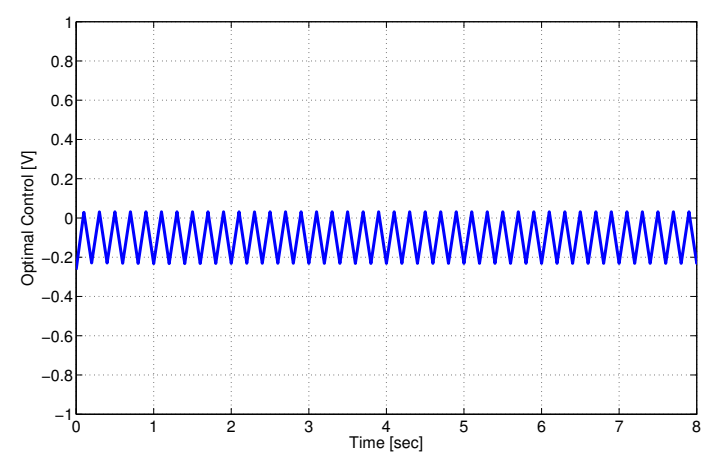

Fig. 7. Optimal Control for Gimbaled System (Case 1)

and the engagement scenario is shown in Fig. 9. The resulting trajectories for the demanded and achieved seeker angles are illustrated in Fig. 10, the optimal control is shown in Fig. 11, and the optimal error is shown in Fig. 12.

In Fig. 10, the solid line denotes the actual (achieved) angle trajectory of the finite-horizon tracking controller, the dashed line denotes the desired seeker angle.

Fig. 9 show that a successful hit is observed with acceptable miss-distance. Comparing these trajectories in Fig. 10, it's clear that the developed finite-horizon SDDRE nonlinear

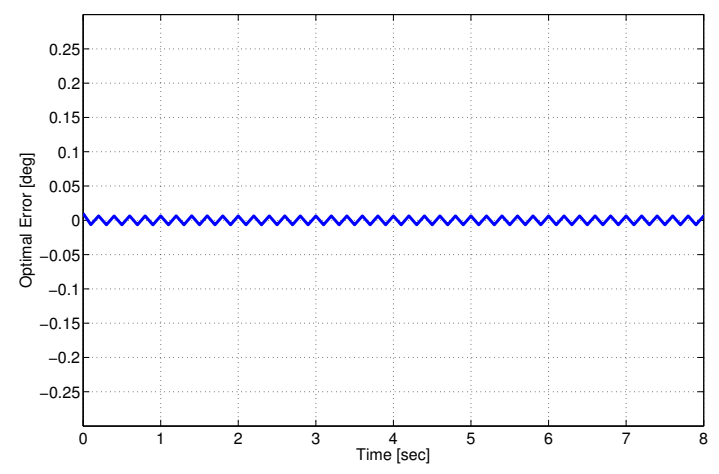

Fig. 8. Optimal Error for Gimbaled System (Case 1) 


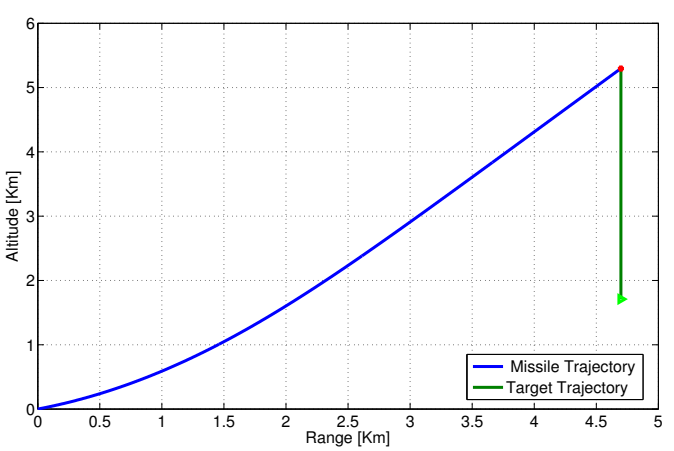

Fig. 9. Missile-Target Engagement Scenario (Case 2)

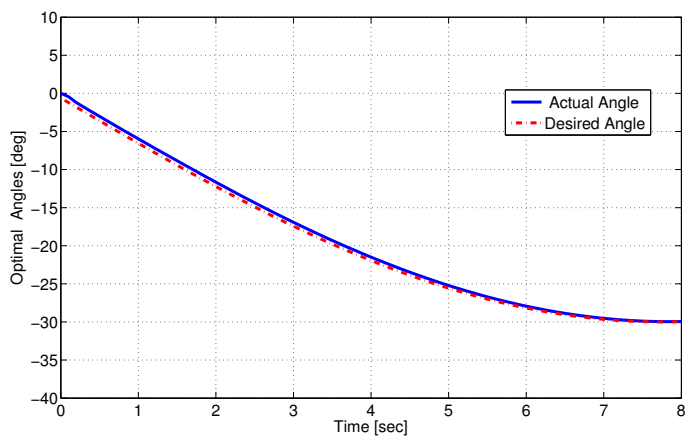

Fig. 10. Angle Trajectories for Gimbaled System (Case 2)

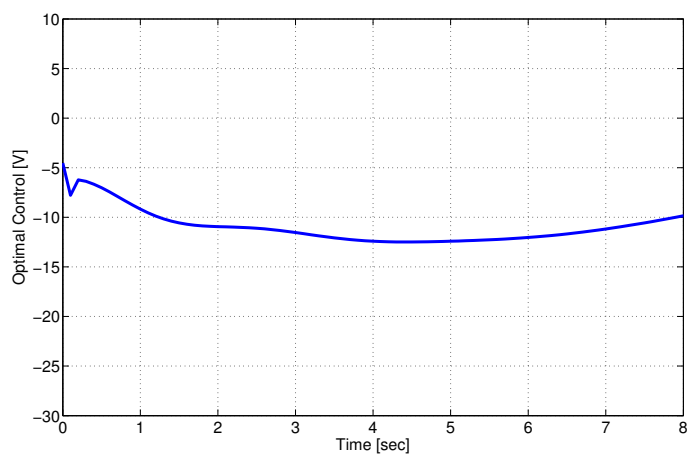

Fig. 11. Optimal Control for Gimbaled System (Case 2)

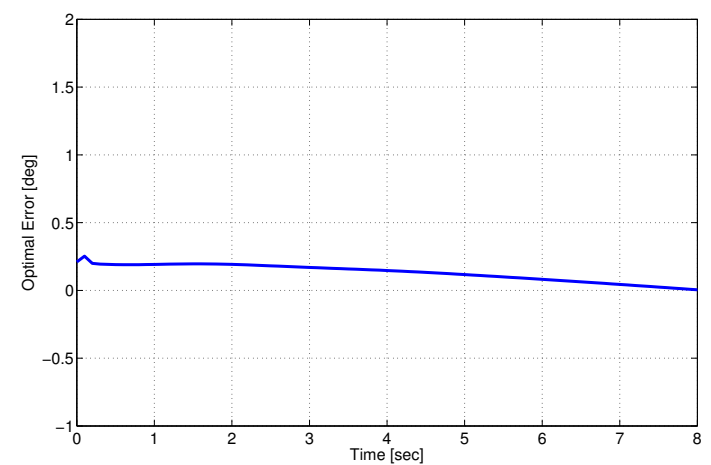

Fig. 12. Optimal Error for Gimbaled System (Case 2)

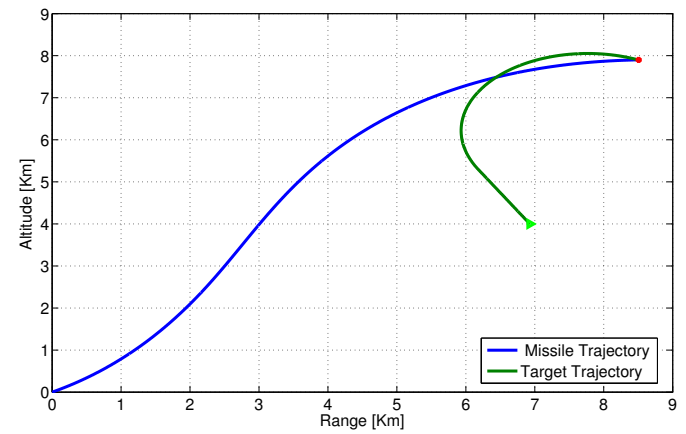

Fig. 13. Missile-Target Engagement Scenario (Case 3)

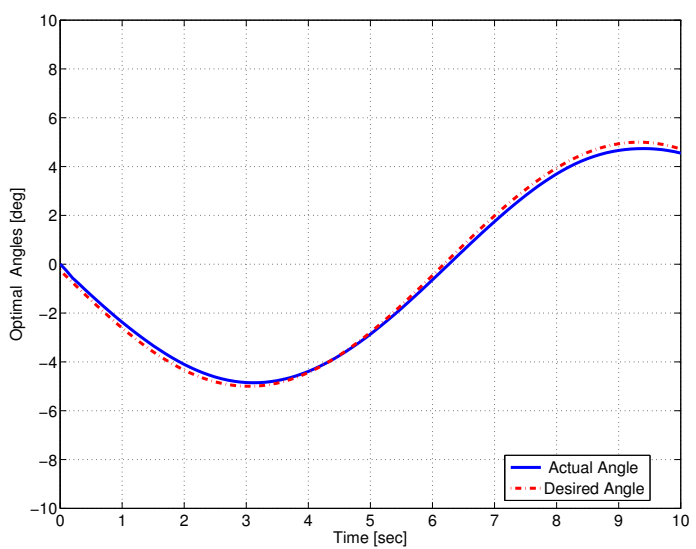

Fig. 14. Angle Trajectories for Gimbaled System (Case 3)

tracking algorithm is able to solve the SDDRE finite-horizon nonlinear tracking problem with an reasonable standard deviation error of $0.075^{\circ}$.

\section{Case 3: Maneuvering Target}

Consider a highly maneuvering target. The simulations were performed for final time of 10 seconds, and the engagement scenario is shown in Fig. 13. The resulting trajectories for the demanded and achieved seeker angles are illustrated in Fig. 14, the optimal control is shown in Fig. 15, and the optimal error is shown in Fig. 16.

In Fig. 14, the solid line denotes the actual (achieved) angle trajectory of the finite-horizon tracking controller, the dashed line denotes the desired seeker angle.

Fig. 13 show that a successful hit is observed with acceptable miss-distance. Comparing these trajectories in Fig. 14, it's clear that the gimbaled seeker performing a very good tracking for the target even when the target tried to make high maneuver. The gimbaled seeker controlled by the developed algorithm is able to track maneuvering target with standard deviation error of $0.026^{\circ}$, which is accepted with this high maneuver.

\section{CONCLUSIONS}

The paper offered a new finite-horizon tracking technique for nonlinear systems. This technique based on change of 


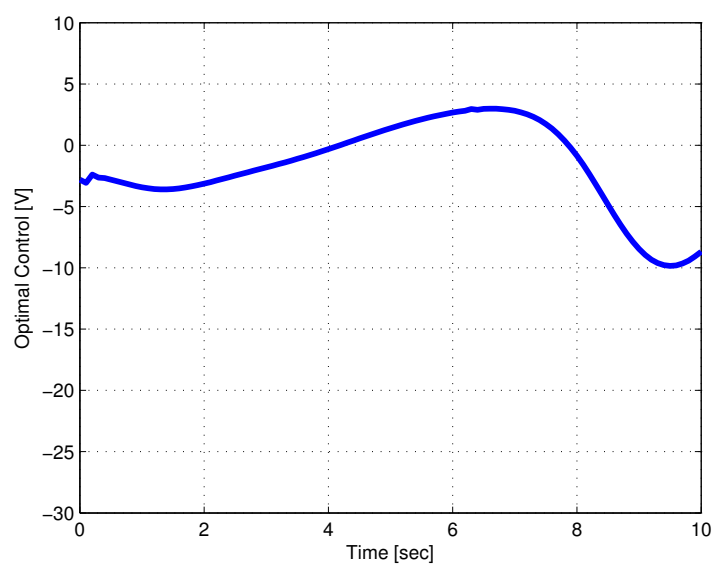

Fig. 15. Optimal Control for Gimbaled System (Case 3)

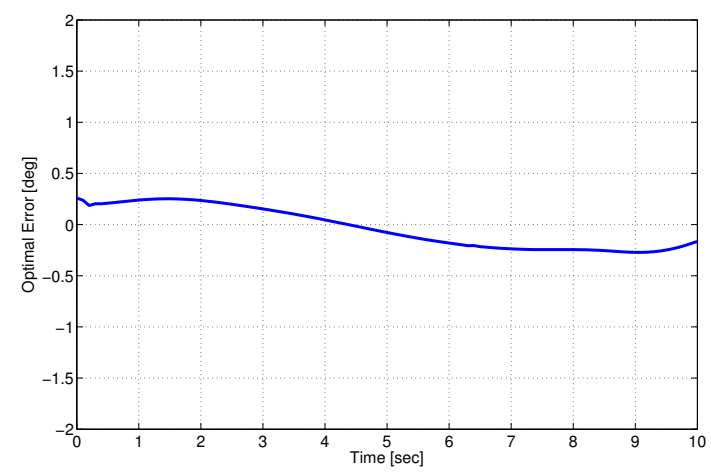

Fig. 16. Optimal Error for Gimbaled System (Case 3)

variables that converts the differential Riccati equation to a linear Lyapunov equation.The Lyapunov equation is solved in a closed form at the given time step. Simulation results for gimbaled system in missile seeker are included. Three engagement scenarios including fixed target, non-maneuvering target, and maneuvering target are considered to demonstrate the effectiveness of the developed technique.

\section{REFERENCES}

[1] C. A. Woolsey, "Review of marine control systems: Guidance, navigation, and control of ships, rigs and underwater vehicles," Journal of Guidance, Control, and Dynamics, vol. 28, no. 3, pp. 574-575, 2005.

[2] G. M. Siouris, Missile Guidance and Control Systems. Springer, 2004.

[3] T. Çimen, "Recent advances in nonlinear optimal feedback control design," Proceedings of the 9th WSEAS International Conference on Applied Mathematics, Istanbul, Turkey, pp. 460-465, May 2006.

[4] J. R. Cloutier, "State-dependent Riccati equation techniques: An overview," Proc. American Control Conference, vol. 2, pp. 932-936, 1997.

[5] T. Cimen, "Development and validation of a mathematical model for control of constrained nonlinear oil tanker motion," Mathematical and Computer Modeling of Dynamical Systems, vol. 15, no. 1, p. 1749, 2009.

[6] A. Ratnoo and D. Ghose, "State-dependent Riccati-equation-based guidance law for impact-angle-constrained trajectories," Journal of Guidance, Control, and Dynamics, vol. 32, no. 1, pp. 320-326, 2009.

[7] S. Vaddi, P. K. Menon, and E. J. Ohlmeyer, "Numerical state-dependent Riccati equation approach for missile integrated guidance control," Journal of guidance, control, and dynamics, vol. 32, no. 2, pp. 699703, 2009.

[8] T. Çimen, "A generic approach to missile autopilot design using statedependent nonlinear control," in 18th IFAC World Congress, 2011, pp. 9587-9600.

[9] A. Heydari and S. N. Balakrishnan, "Path planning using a novel finite-horizon suboptimal controller," Journal of Guidance, Control, and Dynamics, pp. 1-5, 2013.

[10] T. Nguyen and Z. Gajic, "Solving the matrix differential Riccati equation: a Lyapunov equation approach," IEEE Trans. Automatic Control, vol. 55, no. 1, pp. 191-194, 2010.

[11] J. Nazarzadeh, M. Razzaghi, and K. Nikravesh, "Solution of the matrix Riccati equation for the linear quadratic control problems," Mathematical and Computer Modelling, vol. 27, no. 7, pp. 51-55, 1998.

[12] A. Khamis and D. Naidu, "Nonlinear optimal tracking using finitehorizon state dependent Riccati equation ( SDRE)," Proceedings of the 4th International Conference on Circuits, Systems, Control, Signals (WSEAS), pp. 37-42, August 2013, valencia, Spain.

[13] D. S. Naidu, Optimal Control Systems. CRC Press, 2003.

[14] Z. Gajic and M. Qureshi, "The Lyapunov matrix equation in system stability and control," New York: Dover Publications, 2008.

[15] A. Barraud, "A new numerical solution of $x d o t=a 1 * x+x * a 2+d, x(0)=c$," IEEE Transaction on Automatic Control, vol. 22, no. 6, pp. 976-977, Dec. 1977.

[16] A. Khamis, "Design and realization of hardware in loop simulation for a homing guided missile," Ph.D. dissertation, Military Technical Collage, Cairo, 2007.

[17] B. Etkin and L. D. Reid, Dynamics of Flight: Stability and Control. Wiley New York, 1982.

[18] A. Calise, S. Lee, and M. Sharma, "Direct adaptive reconfigurable control of a tailless fighter aircraft," AIAA Guidance, Navigation, and Control Conference, pp. 88-97, 1998.

[19] Y. Ulybyshev, "Terminal guidance law based on proportional navigation," Journal of Guidance, Control, and Dynamics, vol. 28, no. 4, pp. 821-824, 2005. 